\title{
A Novel Approach to Analyze Cross-Cultural Communication Based on New Media Platform and Age
}

\author{
Hao Yu ${ }^{1, \text { a }}$, Jian Zhou ${ }^{2, b^{*}}$, Zongyuan Tan ${ }^{2, \mathrm{c}}$, \\ Zhihong Yang ${ }^{1, d}$ and Wanchun Liu ${ }^{2, e}$ \\ ${ }^{1}$ College of Journalism and Communication, Northwest Minzu University, Lanzhou, China \\ ${ }^{2}$ College of Electrical Engineering, Northwest Minzu University, Lanzhou, China \\ a34414677@qq.com, bzhoujianfrank@126.com, ' $1041689460 @ q q . c o m$, \\ dyangjh@yeah.net, e1054828395@qq.com \\ ${ }^{*}$ The Corresponding author
}

Keywords: Cross-Culture; Communication; Media; Complex Network

\begin{abstract}
This article presents a novel approach to analyze cross-cultural communication based on dynamical complex network nodes. Through the analysis of the data, it is worth remarking that any single nodes of the communication networks could not explain how the information and culture active and motive the awareness of public. Overall, a novel approach is proposed, although its reasonability remains tested, the effectiveness of the cross-cultural communication could be improved.
\end{abstract}

\section{Introduction}

Mutual culture is the key to open the communication. New media provides an unprecedented possibility for the dissemination and enrichment of social culture: it provides a powerful driving force for cultural transmission as part of postmodern culture [1-2]. This paper examines the cultural nature of new media technology and its cultural transmission from the perspective of new media function the paper points out that through the development and application of new media technology [3], we should strengthen the adapting combination of new media resources and combine the technical force to the new media resources, and put forward the development of the new media resources. Promote resource integration and institutional reorganization and other strategies to achieve the effective promotion of cultural transmission [4].

Information is the foundation of social order established, the main form include interpersonal trust, social trust and political trust mass media of various forms of trust have varying degrees of impact [5], in the past affect the relationship between media use and political trust, social trust the study involved more than a study through questionnaires, trying to explore the use of different media and media inherent dependence and trust evaluation newspapers, television, Internet, mobile phones, etc. affect the relationship [6]. the study found, trust in terms of evaluation, compared with social trust, political trust, interpersonal trust is currently still dominate in social trust in China; in terms of media use and trust evaluation, regardless of the impact on interpersonal trust, social trust and political trust evaluation, networks, mobile phones and other new media on trust evaluation is much higher than as television and newspaper traditional media [7], but in terms of media dependent, newspapers, television, Internet, mobile media rely significantly related to interpersonal trust, social trust variable indexes no, but was significantly correlated with political trust Moreover, for interpersonal trust. And social trust evaluation, the use of variables in the media to predict the evaluation of trust over medium dependent variables predictive power of trust evaluation; and for the evaluation of political trust, media dependent variables predictive power of trust in the media far more than the use of evaluation[8-9].i

Alternatively, under certain situation, Chinese cross-cultural is mainly determined by three factors, which are region, network and effectiveness [10]. These new ideas offered a new angle for continuing and improving the further development of the new media culture. In this sense, it is 
clearly that the Chinese cross-cultural has a greater impulse for enhancing the effectiveness of media network. New media is a new type of memory of ethnic minority culture, which is conducive to the preservation and inheritance of minority culture [10-11]. Network media has a huge storage function, the network storage almost does not take up physical space, stored in the network information can be quickly and easily spread through the network, promote the development of ethnic minority culture [12-13].

New media can promote the rapid and widespread spread of ethnic culture. New media is built on the basis of digital technology and network technology, it extends out of the various media forms compared with the traditional media, there are hypermedia, interactive and cross-time and other characteristics, so the new media can fasterly send information to anyone in the world who owns the network [14]. In recent years, in the remote areas of ethnic minorities, those long-term oral propaganda or ritual heritage of the minority culture, under the influence of new media forces, has been fast, broad and effective dissemination.

\section{Culture Network and Media}

In the post-industrial era, with the development of media technology, the rise of the Internet, and the subsequent emergence of a variety of new forms of media, cultural paradigm has changed dramatically, and even bring a new cultural point. The technical determinism in the context of communication research advocates that new media technology is the driving force of social change and the progress of civilization, which is achieved through the dissemination of technical means and representative communication. In fact, the new communication technology does improve the ability of human control of space, reducing the time to pass information, such as printing to solve the problem of rapid production, radio and television is to solve the problem of large-scale rapid release. Specially, the traditional media events are concerned with the social and psychological state based on the consensus culture, but the social and psychological state of the new media events is much more complex, especially in the Chinese social context.

The average path length of effectiveness of new media is given by the following form:

$$
L \sim \frac{\ln N}{\ln \ln N}
$$

Note that this L actually is not very large, implying that the BA scale-free network model also has a certain small-world network feature-two randomly chosen nodes are connected by a fairly short path.

Subsequently, the average node degree of the BA network is approximately equal to $2 \mathrm{~m}$, and the node-degree distribution is approximately given by a power-law form:

$$
P(k) \sim 2 m^{2} k^{-3}
$$

Also, at every step new edges have been added, so the total node degree of the network at time t. Thus, since the number of nodes at this moment is t. The average node degree is approximately equal to $2 \mathrm{~m}$, the above equal to $2.4 \mathrm{~m}$, as clearly shown in Fig. 1 and Table 1.

Table 1 Total number of additions of the Cross-communication

\begin{tabular}{|c|c|c|c|c|}
\hline Degree & Number of add & Cross-degree & Effectiveness & Nodes \\
\hline 170 & 10020 & $\mathrm{H}$ & $71.2 \%$ & 20 \\
\hline 350 & 8000 & $\mathrm{M}$ & $55.3 \%$ & 15 \\
\hline 500 & 900 & $\mathrm{~L}$ & $32.8 \%$ & 5 \\
\hline
\end{tabular}

Many network-based models have been developed and analyzed for studying human opinion dynamics: their modeling, simulation and analysis. The aim is to reveal the underlying mechanism of human opinion formation, evolution and convergence, hoping to gain a better understanding of human social communication behaviors particularly their opinion dynamics. However, these studies 
have not been able to explain the dissemination of new media events and the role of the social factors to explain thoroughly, another reason is that the new media events are subject to historical and cultural context, geographical environment and communication environment, so some new media events only have the cultural particularity in the Chinese context. However, a considerable number of new media events in Chinese communication academics are still using Western theory as an explanatory tool.

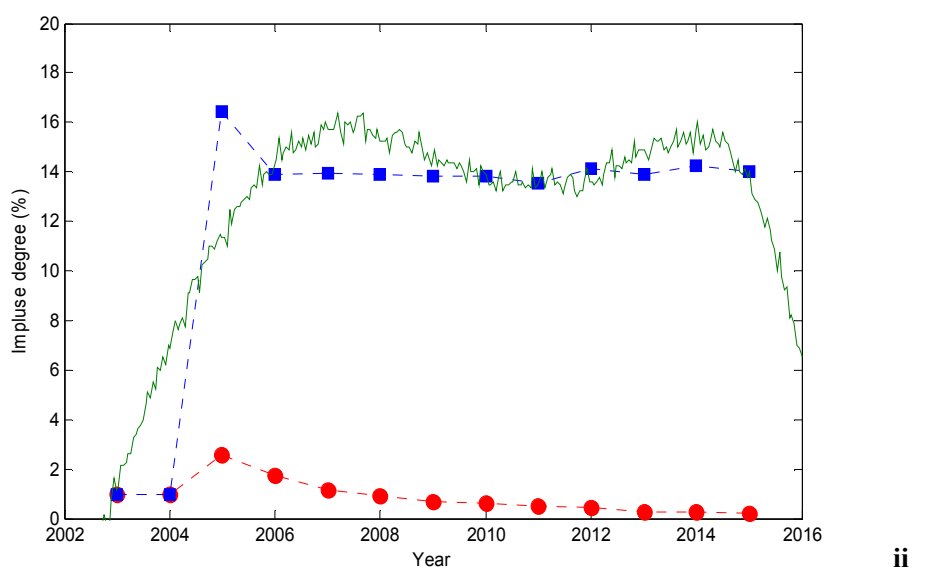

Figure 1. Finite Relation between impulse-degree and year

It is obviously find that emphasizing how cultural and other social forces intertwined to produce a subtle constructive effect on social life. It is also worth remarking that this shift may be of greater inspiration for new media events, as seen in Table 2.

Table 2 Results for the models robustness

\begin{tabular}{|c|c|c|c|c|}
\hline & Type & Network & Culture degree & MLW \\
\hline www.edu & Undirected & Film & 2.23 & $1.7 \mathrm{E}+07$ \\
\hline www.sina & Undirected & Actors & 2.33 & $1.7 \mathrm{E}+08$ \\
\hline www.tencent & Undirected & Actors & 2.81 & $1.7 \mathrm{E}+08$ \\
\hline www.google & Directed & Film & 24.46 & $2.99 \mathrm{C}$ \\
\hline
\end{tabular}

Through comparing the robustness of the random collected data and the network type in the sense described accurately, their maintenance of connectivity against node-removal. As well commonly known, there are three accepted mainstream research paradigms, social science, critical theory and interpretive paradigm. New media research as a relatively independent research point in the study of communication, also in the three kinds of research paradigm on the basis of epistemology and methodology. Chinese government should produce the feasible measures and profitable policies to achieve the ideal goal of the cultural communication. To the social science paradigm at the media level, to explore the new media access, adoption and use and audience awareness, attitudes and behavior between the complex relationship. Under the common background of globalization and localization, how should new media research of Chinese communication academy develop.

It is easily to find that traditional audience research in the new media era suffered unprecedented challenges, the audience of the mass communication era is more aggregated, and the audience of the new media era is more focused and personalized. Although audience-centered research is still quite popular in new media research, user-centered research has also experienced significant growth in recent years, and more scholars have shifted from research to behavioral research. In this sense, any Internet users can not complete the data to modify, delete and add, and the network storage of national cultural information can make users more convenient and quick to retrieve and use, to achieve the sharing of national cultural resources, media content affects people's awareness and 
attitudes, but because of the emergence of new technologies, scholars began to pay attention to how users take the initiative to select some content and ignore other content.

\section{Conclusion}

Through the new media to achieve cross-cultural communication, practice national culture to go out of the strategy. As cross-cultural communication there is a communication barrier, we can through two ways to achieve the cross-cultural transmission of national culture. One is through some Chinese newspapers overseas version and online version of cross-cultural communication. The other is the use of non-textual cultural symbols for the visual transmission, such as through dance performances, folk songs broadcast live, recording video to spread, to eliminate different cultural background of the individual, group or organization in the language exchange formed on the " Heterogeneous "obstacles, to achieve different cultural background between people, between ethnic and national, between countries and countries of mutual understanding and mutual recognition, which is more conducive to the realization of cross-cultural transmission of national culture. The government should produce several profitable policies and feasible measures for improving the effectiveness of the culture communication under the new media circumstances.

Furthermore, from the spread of the scope of view, ethnic minority areas of ethnic culture there is a serious regional limitation, narrow range of communication, did not go out, limited to the national, domestic communication. In other words, from above proposed, we find that only enhance the awareness of public of culture, we will create a "clear" situation for Chinese culture communication. And thus promote the improvement of the quality of the entire national culture.

\section{Acknowledgements}

This work is supported by Fundamental Research Funds for the Central Universities (Grant 31920160003), and by Research Funds for New Silk Road Economic Belt of Northwest Minzu University (Grant xsczl201602).

\section{References}

[1] J. Ruotsalainen, S. Heinonen, Media ecology and the future ecosystemic society, European Journal of Futures Research, Vol. 3 (2015) No.1, pp.1-10.

[2] S.A. Park, J. Kim, Social categorization and cross-cultural exploration of the third-person effect: Perceived impact of North Korea's nuclear test on the self and comparison targets, Studies in Communication Sciences, Vol. 13 (2013) No.1, pp.50-57.

[3] A. Lancichinetti, S. Fortunato, Community detection algorithms: A comparative analysis. Physical Review E, Vol. 80 (2009) No.5, 056117.

[4] K. Sznajd-Weron, Sznajd model and its applications, Acta Physica Polonica B, Vol. 36 (2005) No.8, pp.2537-2547.

[5] M. E. Newman, The structure of scientific collaboration networks, The National Academy of Sciences of the United States of America, 2001, Vol. 98 (2001) No.2, pp.404-409.

[6] N. Cai, J. Cao, H. Ma and C. Wang, Swarm stability analysis of nonlinear dynamical multi-agent systems via relative Lyapunov function, Arab. J. Sci. Eng., 39 (2014), pp.2427-2434.

[7] D.B. Chen, M.S. Shang, Z.H. Lv and Y. Fu, Detecting overlapping communities of weighted networks via a local algorithm, Physica A: Statistical Mechanics and its Applications, Vol. 389 (2010) No.19, pp.4177-4187. 
[8] B.L. Hu, J. Ma and J. Tang, Selection of multiarmed spiral waves in a regular network of neurons, Plos One, Vol. 8 (2013) No.7, e69251.

[9] I.X.Y. Leung, H. Pan, P. Lio and J. Crowcroft, Towards real-time community detection in large networks, Physical Review E, Vol. 79 (2009) No.6, 066107.

[10]J.X. Xi, M. He, H. Liu and J.F. Zheng. Admissible output consensualization control for singular multi-agent systems with time delays, Journal of the Franklin Institute-Engineering and Applied Mathematics, Vol. 353 (2016) No.16, pp.4074-4090.

[11]J. Park, Y.M. Baek and M.Y. Cha, Cross-cultural comparison of nonverbal cues in emoticons on twitter: evidence from big data analysis, Journal of Communication, Vol. 64(2014), No.2 pp.333-354.

[12]T.C. Russo, J. Koreten, Prestige, centrality and learning: A social network analysis of an online class, Communication Education, Vol. 54 (2005) No.3, pp.254-261.

[13]G. Palla, I. Derenyi, I. Farkas and T. Vicsek, Uncovering the overlapping community structure of complex networks in nature and society, Nature, Vol. 435 (2005) No.7340, pp.814-818.

[14]N. Cai, C. Diao and M.J. Khan, A novel clustering method based on quasi-consensus motions of dynamical multi-agent systems, Complexity, 2017, 4978613. 